\section{Making the most of the host}

\section{By Tracey Baas, Senior Editor}

Although recent attention in the HCV space has focused on positive FDA panel recommendations for the protease inhibitors telaprevir and Victrelis boceprevir from Vertex Pharmaceuticals Inc. and Merck \& Co. Inc., respectively, ${ }^{1}$ a French-led academic group has now uncovered targets on host cells that could also be inhibited to impede HCV entry. ${ }^{2}$

The new targets include a pair of receptor tyrosine kinases-epidermal growth factor receptor 1 (EGFR1; HER1; ERBB1) and EPH receptor A2 (EPHA2) - that are inhibited by the marketed cancer drugs Tarceva erlotinib and Sprycel dasatinib (see Figure 1, "Host factors involved in HCV entry and their potential as therapeutic targets").

The team, led by Thomas Baumert, began to suspect that tyrosine kinase inhibitors (TKIs) could have antiviral activity in humans after University Hospital Pontchaillou researchers published a case study in which a hepatocellular carcinoma patient cleared his HCV infection after receiving erlotinib. ${ }^{3}$

Baumert is a professor of medicine at the University of Strasbourg and head of the Strasbourg Virology Unit U748 at the Institut National de la Santé et de la Recherche Médicale (INSERM).

Using a functional small interfering RNA kinase screen, Baumert's team identified 58 kinases that affected the HCV life cycle, including EGFR1 and EPHA2.

In vitro, the EGFR1 inhibitor Tarceva and the EPHA2 inhibitor Sprycel blocked entry, infection and cell-to-cell transmission of different HCV genotypes compared with vehicle.

Tarceva, from Astellas Pharma Inc. and partner Roche, is marketed to treat pancreatic cancer and non-small cell lung cancer (NSCLC) and is in Phase III testing for liver cancer. Sprycel, from Bristol-Myers Squibb Co. and partner Otsuka Pharmaceutical Co. Ltd., is marketed to treat acute lymphoblastic leukemia (ALL) and chronic myelogenous leukemia (CML) and is in Phase III trials for prostate cancer and Phase II testing for breast cancer.

The two drugs inhibited both the major genotypes of HCV and diverse strains of the virus. In addition, the drugs were HCV specific and did not inhibit other viruses.

In chimeric mice infected with HCV genotype 2a, Tarceva decreased HCV RNA levels by more than $90 \%$ compared with placebo. The drug was given 5 days before HCV infection and for 20 days thereafter. After treatment stopped, viral load reached levels similar to those seen with placebo treatment.

Data were published in Nature Medicine.
"The antiviral activity of erlotinib—and likely other inhibitors of EGFR1-across different HCV genotypes holds the potential for being useful for HCV-infected patients with diverse viral genotypes," said Thomas Pietschmann, an author on the paper and head of the Department of Experimental Virology at Twincore. According to Sam Hopkins, CSO of Scynexis Inc., the $90 \%$ reduction in viral load was modest but not unexpected given that treatment only lasted for about 3 weeks.

He noted that the mouse model used by the researchers "is one of the better models that the field of HCV research has to offer at this point in time because it provides the advantage of recreating human hepatitis $\mathrm{C}$ infection in human hepatocytes in a murine tissue/host background."

Hopkins added: "The best way to demonstrate activity against a human protein target relies on demonstrating antiviral activity in an infected human cell where activity is clearly dependent on expression of the human host protein cofactor."

Scynexis has a host factor-targeting cyclophilin inhibitor in Phase I testing for $\mathrm{HCV}$.

Kai Lin, virology group head of the HCV effort at Novartis AG, said he wanted to see if the TKIs work after infection takes hold. Lin also wanted to see HCV-infected chimeric mice treated with an EGFR1 inhibitor in combination with other antivirals.

Similarly, Edouard Bardou-Jacquet, the clinician who led the Pontchaillou case study and is now at Rennes University Hospital's liver unit, said he was "very interested to see the cumulative effect of erlotinib with protease inhibitors-boceprevir or telaprevir-on chimeric mice infected with genotype 1 and 4 viruses and moreover with protease inhibitor-resistant virus."

\section{Setting the standard}

The current standard of care for HCV, pegylated interferon- $\alpha$ and ribavirin, is poorly tolerated and only works in about half of genotype 1-infected patients. The emerging consensus is that an HCV NS3 protease inhibitor will join the cocktail as standard of care, but down the line there are a handful of next-generation compounds in development that seek to take interferon and ribavirin out of the equation entirely. ${ }^{4}$

"If the first wave of non-interferon/ribavirin HCV therapies is represented solely by the direct-acting antivirals, the next wave should also include the host-targeting antivirals," said Flossie Wong-Staal, professor emeritus of medicine at the University of California, San Diego and EVP and CSO of infectious disease company iTherX Pharmaceuticals Inc.

Host-targeting antivirals in the clinic for HCV include two Novartis compounds. Its Phase III alisporivir cyclophilin inhibitor is the most advanced. The pharma's other compound is NIM811, which is in Phase II studies.

iTherX's entry inhibitor is ITX 5061, which targets the scavenger receptor class B member 1 (SCARB1) co-receptor. It is in Phase II testing.

Compared with viral targets, attacking host factors has the advantages of broad genotype coverage and a lower likelihood of resistance. "The greatest concern about host-targeting antivirals is safety," Wong-Staal said. 
Figure 1. Host factors involved in HCV entry and their potential as therapeutic targets. HCV associates with very low-density and low-density lipoproteins (LPs) in the bloodstream, allowing the virus to bind to hepatocytes through glycosaminoglycans (GAGs) and the low-density lipoprotein receptor (LDLR) [a]. Engagement by the virus of scavenger receptor class B member 1 (SCARB1), CD81, claudin 1 (CLDN1) and occludin (OCLN) [b] leads to endocytosis of virions [c]. HCV fuses with acidified endosomes and releases its genome into the cytosol [d].

Activation of the tyrosine kinase receptors epidermal growth factor receptor (EGFR1; HER1; ERBB1) or EPH receptor $\mathrm{A} 2$ (EPHA2) has been suggested by Lupberger et al. to regulate CD81-CLDN1 interactions and membrane fusion, which are essential for HCV entry. Erlotinib and dasatinib were shown to inhibit HCV entry by interfering with the CD81-CLDN1 association by an as yet unknown mechanism.

Figure modified from Gerold et al. ${ }^{6}$

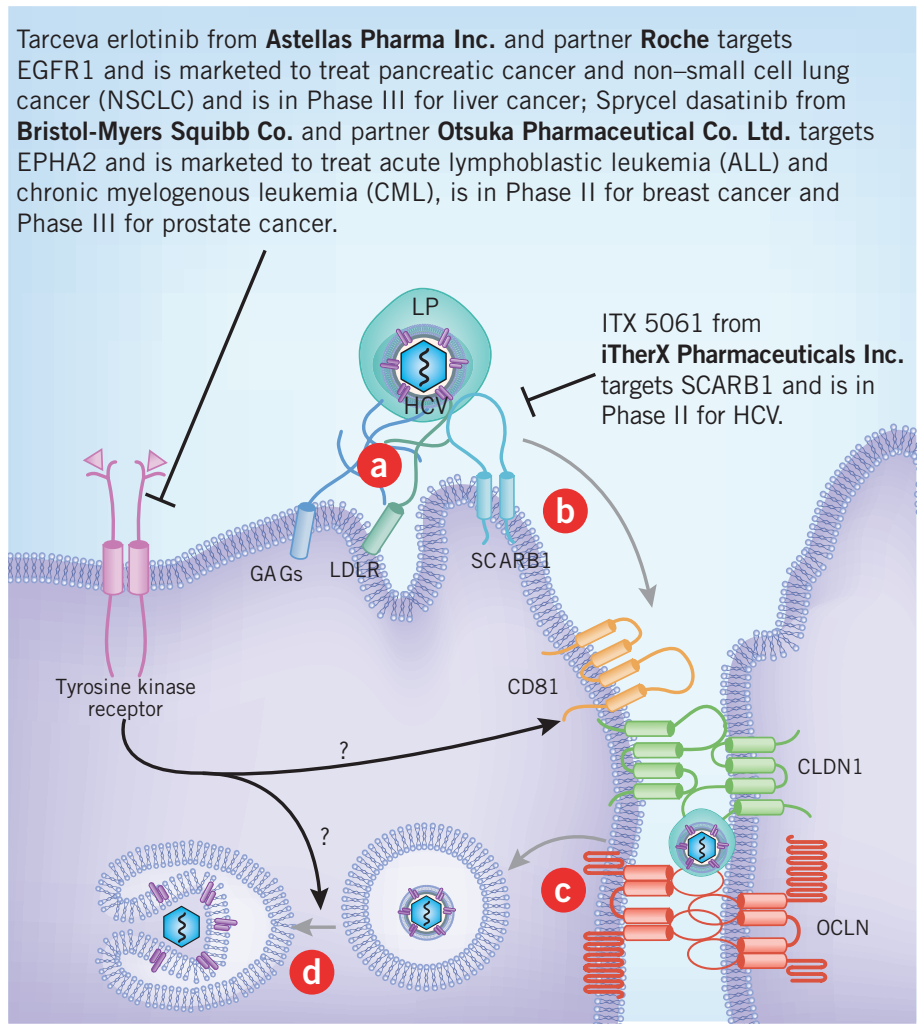

She was echoed by Raymond Chung, director of hepatology at Massachusetts General Hospital and Harvard Medical School. "Using host-targeting antivirals, you're going to have to find the sweet spot where dosing is toxic to the virus with limited toxicity to the host," he said.

In 2009, Chung's laboratory identified phosphatidylinositol 4-kinase catalytic $\alpha$ (PI4KA) as an HCV-replication host cofactor. ${ }^{5}$ His group is screening and developing PI4KA inhibitors.

Ivan Horak, CSO and CMO at Symphogen A/S, said adding a host-targeting agent to standard of care for HCV could circumvent the virus' rapid mutation. However, he thinks "the drug-drug interaction in such a cocktail is going to be a major problem."

Symphogen's Sym004, a mAb that targets the extracellular domains of EGFR1, is in Phase I/II trials for cancer.

Although drug-drug interactions could be an issue, Lin thinks molecules that block host cell cofactors of HCV could be particularly useful in infected patients who receive liver transplants. He said the overarching goal would be to use drugs that target the virus before transplantation, followed by an EGFR1-targeting drug after transplant to prevent reinfection.

Bardou-Jacquet wasn't sure about using EGFR1 inhibitors in transplant patients.

"Usually EGFR1 inhibitors are contraindicated for abdominal surgery and need to be stopped, as the risk of perforation and anastomosis leakage is substantial. This will be a major issue in liver transplantation and will need to be carefully assessed," he said. "It may depend on the antibody or molecule used, as sorafenib has been used safely in a small number of liver transplant cases."

Nexavar sorafenib, an inhibitor of multiple receptor tyrosine kinases from Onyx Pharmaceuticals Inc. and Bayer AG, is marketed to treat liver cancer and renal cancer. The drug also is in Phase III testing for thyroid cancer and NSCLC and Phase II trials for colorectal and ovarian cancer.

\section{Playing host}

Hopkins noted that erlotinib "exhibits relatively low antiviral potency and is clearly not optimized to treat HCV." But he thinks Baumert's group is now in a prime position to run its own drug discovery studies.

"Many of the tools that they used to vet the target now can be used to support an intensive medicinal chemistry program with the goal of identifying more potent and specific inhibitors of receptor kinase activity than erlotinib to treat HCV," Hopkins said.

$\mathrm{He}$ also suggested that the academics would be able to move into human studies.

"Since there is a long clinical history with erlotinib, it is conceivable that these researchers could conduct limited, investigator-initiated clinical studies to address the feasibility of this approach in selected 


\section{ANALYSIS}

patients with hepatocellular carcinoma and chronic HCV infection," said Hopkins. "I think the main issue will be demonstrating the absence of harmful effect in patients without cancer. Chronic HCV patients have longer-term survival compared with many cancer patients. The doses and dose scheduling will most likely be different."

Baumert told SciBX that his group plans to conduct Phase I safety studies of erlotinib in patients with chronic HCV infection and liver disease.

Baumert's findings are patented and available for licensing through INSERM Transfert S.A., the private tech transfer subsidiary of INSERM. His team is also working to develop humanized antibodies that target receptor tyrosine kinases.

Based on the work conducted in Baumert's laboratory, INSERM Transfert also plans to spin off and support an antiviral company that will focus on the development of new therapeutic approaches in hepatitis $\mathrm{C}$ and other viral diseases.

Baas, T. SciBX 4(19); doi:10.1038/scibx.2011.530

Published online May 12, 2011

\section{REFERENCES}

1. McCallister, E. BioCentury 19(19), A12-A15; May 2, 2011

2. Lupberger, J. et al. Nat. Med.; published online April 24, 2011; doi:10.1038/nm.2341

Contact: Thomas F. Baumert, University of Strasbourg,
Strasbourg, France

e-mail: thomas.baumert@unistra.fr

3. Bardou-Jacquet, E. et al. Gut 60, 879-880 (2011)

4. McCallister, E. BioCentury 19(19), A12; May 2, 2011

5. Tai, A.W. et al. Cell Host Microbe 5, 298-307 (2009)

6. Gerold, G. \& Rice, C.M. Nat. Med. 17, 542-544 (2011)

COMPANIES AND INSTITUTIONS MENTIONED

Astellas Pharma Inc. (Tokyo:4503), Tokyo, Japan

Bayer AG (Xetra:BAY), Leverkusen, Germany

Bristol-Myers Squibb Co. (NYSE:BMY), New York, N.Y.

Harvard Medical School, Boston, Mass.

INSERM Transfert S.A., Paris, France

Institut National de la Santé et de la Recherche Médicale,

Strasbourg, France

iTherX Pharmaceuticals Inc., San Diego, Calif.

Massachusetts General Hospital, Boston, Mass.

Merck \& Co. Inc. (NYSE:MRK), Whitehouse Station, N.J.

Novartis AG (NYSE:NVS; SIX:NOVN), Basel, Switzerland

Onyx Pharmaceuticals Inc. (NASDAQ:ONXX), Emeryville, Calif.

Otsuka Pharmaceutical Co. Ltd., Tokyo, Japan

Rennes University Hospital, Rennes, France

Roche (SIX:ROG; OTCQX:RHHBY), Basel, Switzerland

Scynexis Inc., Research Triangle Park, N.C.

Symphogen A/S, Copenhagen, Denmark

Twincore, Hannover, Germany

University Hospital Pontchaillou, Rennes, France

University of California, San Diego, La Jolla, Calif.

University of Strasbourg, Strasbourg, France

Vertex Pharmaceuticals Inc. (NASDAQ:VRTX), Cambridge, Mass. 